\title{
A Chemical Chaperone That Prevents Insulin Fibrillation
}

\author{
Anirban Das, ${ }^{[a]}{ }^{[+]}$Yogesh M. Gangarde, ${ }^{[a]}{ }^{[+]}$and Ishu Saraogi ${ }^{*}[\mathrm{a}][\mathrm{b}]$ \\ [a] Department of Chemistry, ${ }^{[b]}$ Department of Biological Sciences, Indian Institute of Science Education and Research \\ Bhopal, Bhopal Bypass Road, Bhauri, Bhopal 462066, MP, India
}

Keywords: aggregation $\bullet$ chemical chaperone $\bullet$ formulation $\bullet$ amyloid $\bullet$ inhibitor

\begin{abstract}
Insulin, a peptide hormone, is susceptible to amyloid formation upon exposure to aberrant physiological conditions, resulting in a loss of its bioactivity. For mitigating insulin aggregation, we report a molecule called PAD-S, which completely inhibited insulin fibril formation, and preserved insulin in its soluble form. Circular Dichroism spectroscopy showed that PAD-S was able to maintain the native structure of insulin, thus acting as a chemical chaperone. Seeded aggregation kinetics suggest that PAD-S inhibited primary nucleation events during aggregation. This is consistent with molecular docking results which suggest that PAD-S binds strongly to native insulin monomers/dimers. Through a competitive binding experiment with 'LVEALYL' peptide, we conclude that PAD-S likely binds to the amyloid prone B11-B17 residues of insulin thereby preventing its aggregation. PAD-S was also effective in disaggregating preformed insulin fibrils to non-toxic species. PAD-S treated insulin was functional as indicated by its ability to phosphorylate Akt. PAD-S was also highly effective in preventing the aggregation of insulin biosimilars. The low cellular cytotoxicity of PAD-S, and amelioration of aggregation-induced toxicity by PAD-S treated insulin further highlights its potential as an effective chemical chaperone.
\end{abstract}

\section{INTRODUCTION}

Insulin, a 51-amino acid peptide hormone, has an important role in glucose metabolism. ${ }^{1}$ Insulin underproduction or insensitivity causes diabetes, a condition in which blood glucose levels are elevated. ${ }^{2}$ Such patients are administered insulin from external sources to control the levels of blood sugar in their body. This has resulted in a large worldwide demand for insulin necessitating the production of insulin on an industrial scale. Interestingly, insulin forms amyloid aggregates upon exposure to conditions like acidic $\mathrm{pH}$ (2-4), elevated temperature, mechanical agitation: conditions frequently encountered during its commercial production. The relative ease of insulin aggregation poses a problem during its industrial production and its pharmaceutical use. ${ }^{3,4}$ The resulting loss of biological activity hinders proper control of glucose levels in patients. Additionally, subcutaneous amyloid plaque formation along with cutaneous allergic reactions have been observed in many diabetic patients at the repeated site of insulin injection. ${ }^{5-7,8}$ Thus, efforts to develop small molecules that specifically inhibit insulin aggregation are of prime importance.

Small organic molecules e.g., polyphenolic compounds, ${ }^{9-13}$ supramolecules, ${ }^{14-16}$ peptide-based inhibitors ${ }^{17-20}$ have been screened for insulin aggregation inhibition activity. However, these molecules suffer from several limitations including lack of specificity, incomplete inhibition at high molar ratios with respect to insulin, proteolytic degradation, and lack of understanding about the mechanism of in- hibition. ${ }^{21}$ We have earlier reported pyridylamide derivatives, which reduced insulin fibrillation to $>80 \%$, and one of them (M1) was successful in keeping insulin in its biologically active form. ${ }^{22,23}$ Here, we report a molecule called PAD$\mathrm{S}$, having a sulfonate group at the terminus of the molecule in addition to hydrophobic side-chain functionalities on the pyridyl amide core present in M1. These modifications were inspired by reports of the presence of sulfonate functionalities on few known inhibitors of insulin aggregation. ${ }^{24,25,26}$ p-sulfonatocalixarene and sulfobutylether- $\beta$-cyclodextrin macrocycles were also proposed to disintegrate insulin fibrils owing to the interaction of negatively charged sulfonates with the positively charged surface of insulin fibrils. ${ }^{15,16}$ We found that PAD-S, containing one sulfonate group completely inhibited insulin aggregation at equimolar ratio. PAD-S acted by inhibiting primary nucleation of insulin in a dose dependent manner. PAD-S was also able to disintegrate preformed insulin fibrils to nontoxic form. PAD-S effectively inhibited aggregation of insulin biosimilars, and preserved the native structure and function of insulin, thus acting as a chemical chaperone. 


\section{RESULTS AND DISCUSSION}

Structure-activity relationship (SAR) studies of PAD-S and its fragments. PAD-S (Figure $1 \mathrm{~A}$ ) was successfully synthesized with a few modifications following the synthetic protocol reported earlier. ${ }^{22,27}$ The extent of insulin fibrillation in the presence of PAD-S was monitored by fluorescence spectroscopy using thioflavin-T (ThT). ${ }^{28}$ We incubated native insulin solution $(15 \mu \mathrm{M}, \mathrm{pH} 1.6 \mathrm{HCl}, 0.1 \mathrm{M} \mathrm{NaCl})$ in the presence of PAD-S and allowed it to aggregate at 60 ${ }^{0} \mathrm{C}$. The extent of insulin fibrillation over time was measured by ThT fluorescence. Insulin treated with a fivefold molar excess of PAD-S did not show any enhancement in ThT fluorescence (Figure 1B). In contrast, significant enhancement of ThT fluorescence was observed in solution containing insulin alone due to the formation of amyloid fibrils. These data indicate that PAD-S likely inhibits insulin fibrillation. To rule out any interference from PAD-S on ThT fluorescence, we mixed $75 \mu \mathrm{M}$ PAD-S (highest concentration used in the ThT assay) with ThT, and the change in fluorescence signal was recorded. A small change $(\sim 10 \%)$ in the ThT fluorescence intensity in the presence of PAD-S ruled out any artefacts due to interference of PAD-S with ThT fluorescence.

To rationalize which molecular features of PAD-S were essential for the inhibitory activity, we tested fragments of PAD-S for insulin aggregation inhibition (Figure 1A). The fragment PAD, lacking the pendant sulfonate, or 3-APS containing only the sulfonate functionality did not have any effect on insulin aggregation (Figure 1B). A small ( 30\%) decrease in ThT fluorescence intensity was observed with the fragment PA-S containing only the lower half of PAD-S. The results of the SAR analysis indicate that the presence of all the key molecular features and their proper spatial organization is required for optimal activity.

PAD-S shows dose dependent inhibition of insulin aggregation. To assess the concentration-dependent effect of PAD-S on insulin aggregation, insulin was treated with different concentrations of PAD-S, and the aggregation kinetics were monitored over time using ThT assay. PAD-S showed dose dependent inhibition of insulin fibrillation with complete inhibition beyond equimolar concentration. The inhibition was a result of delay in the lag phase indicating that PAD-S likely inhibited the primary nucleation of insulin monomers during fibrillation (Figure $2 \mathrm{~A}$ ). These results indicate that PAD-S primarily bound either to the monomer or very early oligomers of native insulin and acted on primary nucleation stage of insulin fibrillation.

PAD-S preserves the native structure of insulin. Insulin is a predominantly $\alpha$-helical protein which upon fibril formation transforms into a $\beta$-sheet structure that has been well characterized by Circular Dichroism (CD) spectroscopy. ${ }^{29}$ To evaluate the effect of PAD-S on insulin aggregation, the insulin-PAD-S complex was monitored by CD before and after $12 \mathrm{~h}$ of incubation at $60^{\circ} \mathrm{C}$. The minima at 208 and $222 \mathrm{~nm}$ in the CD spectrum of insulin before aggregation indicates that insulin predominantly exists in an $\alpha$-helical form (Figure 2B, green). The appearance of a minimum at $218 \mathrm{~nm}$ after $12 \mathrm{~h}$ of aggregation (Figure $2 \mathrm{~B}$, black) signifies $\beta$-sheet structure of insulin due to fibril formation. Insulin treated with equimolar PAD-S largely retained its helical signature (Figure 2B, yellow), although a lower concentration of PAD-S was significantly less effective in doing so
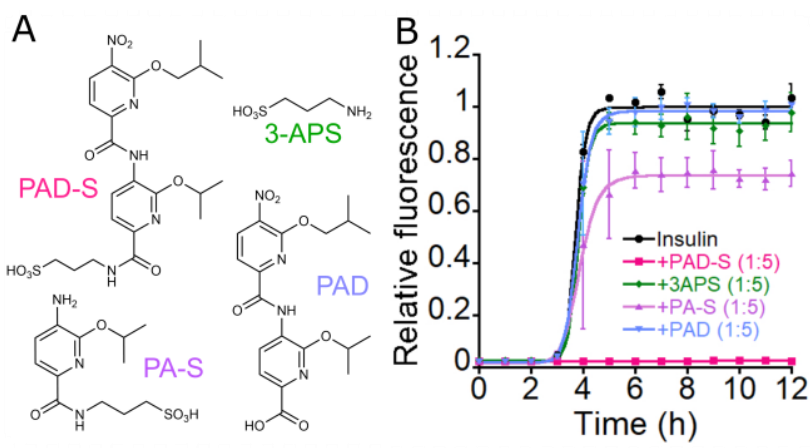

Figure 1. A) Chemical structures of PAD-S and its fragments used in this study. B) Insulin aggregation inhibition with PAD-S and its fragment molecules (insulin:molecule $=1: 5$ ). The error bars indicate standard deviations from three independent experiments. The insulin concentration is $15 \mu \mathrm{M}$ and kinetics were carried out in $\mathrm{pH} 1.6$ at $60^{\circ} \mathrm{C}$.

(Figure 2B, purple). PAD-S alone did not show any structure in the $\mathrm{CD}$ spectrum, and did not alter the native structure of insulin before aggregation. Our results suggest that PAD-S not only inhibits insulin fibrillation, but also preserves the protein in its native helical form, thus acting as a chemical chaperone.

We determined the amount of soluble insulin present after PAD-S treatment using a Bradford assay. PAD-S could successfully maintain the entire protein in its soluble form, comparable to native non-aggregated insulin (Figure 2C). In contrast, the sample containing aggregated insulin in the absence of PAD-S did not contain any soluble insulin. Native PAGE gel electrophoresis revealed that PAD-S treated insulin had a mobility similar to native insulin (Figure 2D) although PAD-S seems to have changed the conformational distribution of insulin. The extra bands in case of PAD-S treated insulin (lane 3) may be attributed to the stabilization of other oligomeric states of insulin, compared to the native form in which predominantly a single band was observed (lane 1). In contrast, insulin aggregated in the absence of PAD-S did not show any distinct bands as a result of aggregation. These results were supported by transmission electron microscopy (TEM) imaging, in which no fibril formation was observed in case of PAD-S treated insulin at equimolar concentration of the molecule (Figure 2E).

Binding of PAD-S to insulin. Insulin contains 4 tyrosine residues at A14, A19, B16, B26 positions which are known to change fluorescence on small molecule binding. ${ }^{30} \mathrm{We}$ used the change in tyrosine fluorescence as a readout to probe the direct binding of PAD-S with insulin. A fixed concentration of insulin $(5 \mu \mathrm{M})$ was titrated with increasing concentrations of PAD-S. Tyr fluorescence decreased with increasing concentration of PAD-S indicating interaction between insulin and the small molecule (Figure $3 \mathrm{~A}$ ). The $\mathrm{K}_{\mathrm{d}}$ was determined to be $14 \pm 3 \mu \mathrm{M}$ between insulin and PAD$S$ (for details, see Materials and Methods). PAD-S did not show any fluorescence when excited at the excitation wavelength of tyrosine, signifying no contribution of PAD-S fluorescence to the tyrosine signal. 


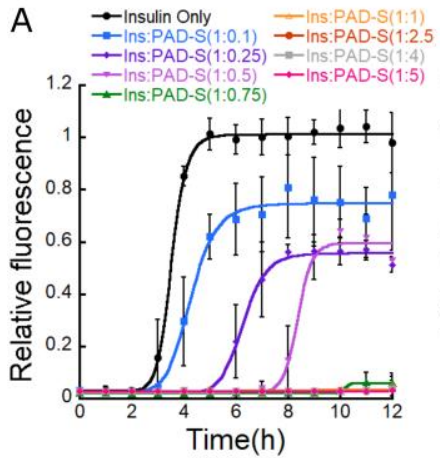

$\mathrm{D}$

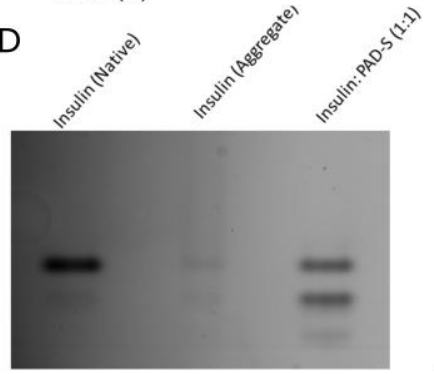

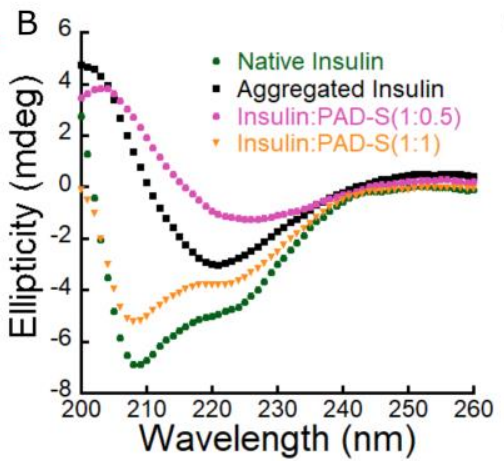

$\mathrm{E}$

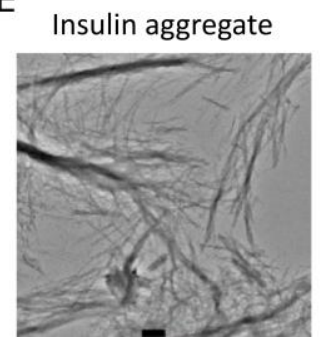



Insulin:PAD-S (1:1)

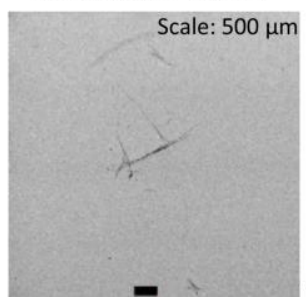

Figure 2. A) Dose-dependent effect of PAD-S on insulin aggregation $\left.\left(15 \mu \mathrm{M}, \mathrm{pH} 1.6,60{ }^{\circ} \mathrm{C}\right) . \mathrm{B}\right) \mathrm{CD}$ spectra of insulin $(3.75 \mu \mathrm{M}$, with or without PAD-S at specific concentrations) at the end of fibrillation. C) Bradford assay showing soluble insulin content at the end of the aggregation in the absence and presence of PAD-S (Ins: PAD-S = 1:0.5 and 1:1 molar ratio) D) Native PAGE image showing inhibition of insulin aggregation by PAD-S. E) TEM images of insulin (with or without equimolar concentration of PADS) at the end of fibrillation. The data and the images are representative of three independent experiments.

Molecular docking studies were carried out to identify potential binding site of PAD-S to monomeric and dimeric insulin (PDB: 1GUJ). The results showed more favorable interaction of PAD-S with the insulin dimer, albeit it also interacted with insulin monomer. The binding energies for the monomer and dimer were found to be -4.0 and -5.9 $\mathrm{kcal} / \mathrm{mol}$ respectively (Figure 3B, 3C). The interacting FFY residues in the $B$ chain of the insulin dimer provides the site that triggers the fibril formation as well as the interacting site for insulin fibril elongation. ${ }^{26,31}$ PAD-S also interacts with the B-chain $\mathrm{C}$ terminal residues, namely ThrB27, ProB28, LysB29, which assist in the self-association and dimerization of the protein. ${ }^{32}$ On the other hand, PAD-S interacts mainly with the monomer in the 'LVEALYLV' region of the insulin B chain, with predominant interaction with
AlaB14, TyrB16, LeuB17, ValB18. Another important interacting residue is HisB10, which is known to play a role in insulin fibrillation. ${ }^{33}$

PAD-S interacts in the B11-B17 'LVEAYL' region to prevent its aggregation. Molecular docking studies reveal that PAD-S interacts with the 'LVEALYL' residues of insulin B chain to prevent its aggregation. Previously, Ivanova et al. and Ballet et al. have reported that sub-stoichiometric amount of this peptide accelerates insulin aggregation. ${ }^{34,35}$ Ivanova et al. have also proposed that this short region in the B chain forms the spine of insulin fibrils. We checked whether PAD-S could inhibit insulin aggregation in the
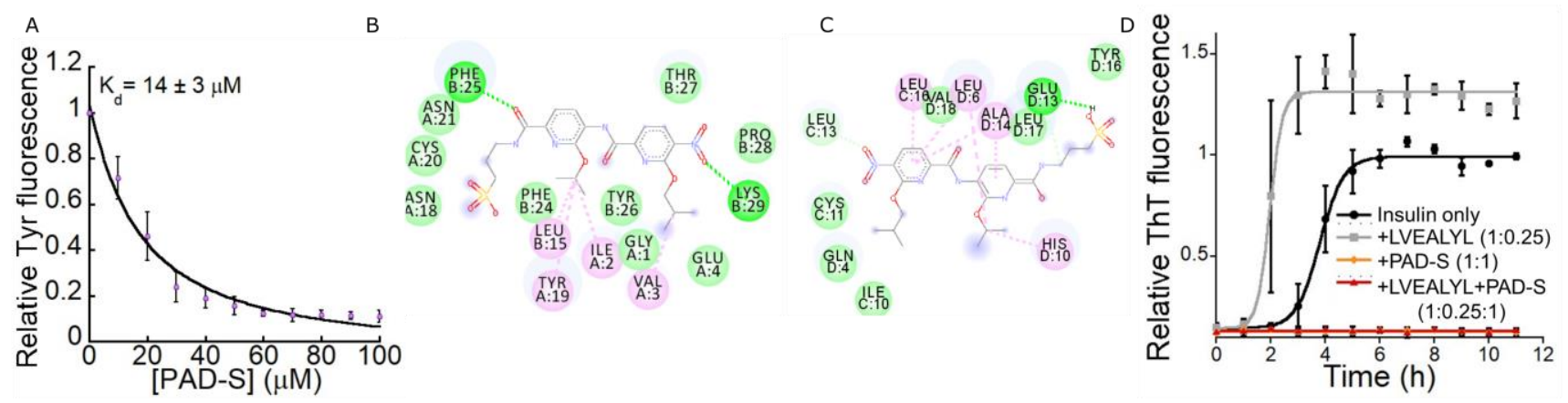

Figure 3. A) Relative change in tyrosine fluorescence ( $\lambda_{\text {ex }}: 275 \mathrm{~nm}, \lambda_{\mathrm{em}}: 303 \mathrm{~nm}$ ) of insulin $(5 \mu \mathrm{M})$ upon titration with PAD-S (0-100 $\mu \mathrm{M})$. The error bars indicate standard deviations from two independent experiments. Interacting residues upon binding of PAD-S to B) insulin dimer (PDB: 1GUJ), C) insulin monomer (PDB: 1GUJ). D) Competition experiment between PAD-S and LVEALYL for binding to insulin. LVEALYL peptide accelerates insulin aggregation; PAD-S inhibits insulin aggregation even in the presence of LVEALYL. 

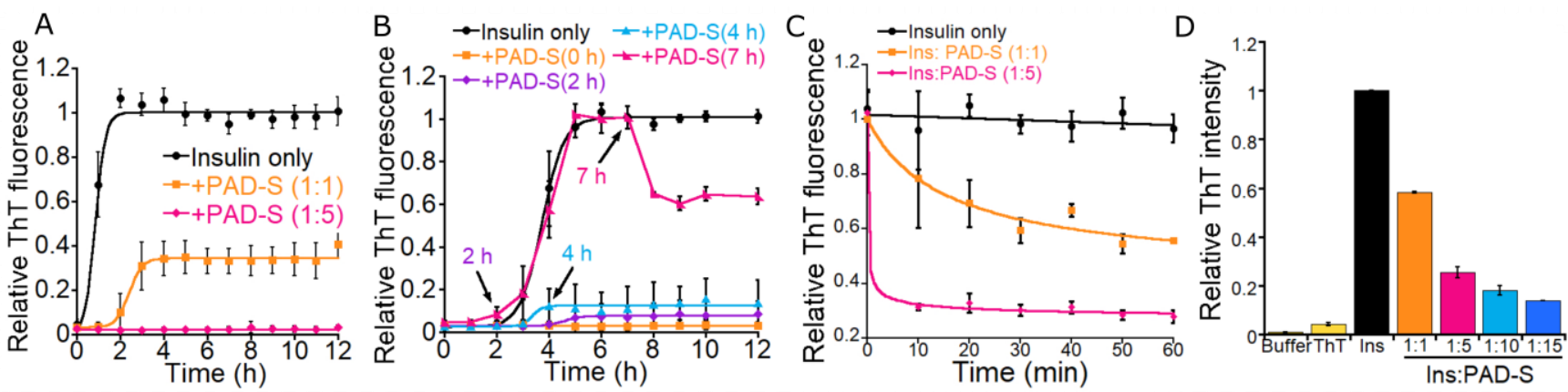

Figure 4. A) Seeded (3\% v/v) aggregation kinetics of insulin $\left(15 \mu \mathrm{M}, \mathrm{pH} 1.6,60^{\circ} \mathrm{C}\right)$ at specified insulin: PAD-S ratio. The error bars indicate standard deviations from three independent experiments. B) Fibrillation kinetics of insulin at different time point addition ( $2 \mathrm{~h}, 4 \mathrm{~h}, 7 \mathrm{~h})$ of equimolar PAD-S. The error bars indicate standard deviations from three independent experiments. C) Time dependent kinetics showing disintegration of mature insulin fibrils by PAD-S at insulin: PAD-S concentration of 1:1 (15 $\mu$ M, pH 1.6, $60{ }^{\circ} \mathrm{C}$ ). Data is average of two independent experiments. D) Final ThT intensity after disintegration of mature insulin fibrils by different concentrations of PAD-S $\left(15 \mu \mathrm{M}, \mathrm{pH} 1.6,60^{\circ} \mathrm{C}\right)$. The ThT data was recorded after $1 \mathrm{~h}$ of agitation. Data is average of three independent experiments.

presence of 'LVEALYL' peptide. We found that insulin aggregation was indeed faster in presence of the peptide, and that PAD-S was successful in inhibiting insulin aggregation both in the absence and presence of the peptide. This data supports that PAD-S outcompetes 'LVEALYL' to bind to insulin and prevent its aggregation (Figure 3D).

PAD-S likely binds to native insulin to prevent its aggregation. We carried out seeded aggregation to explore the mechanism of fibril inhibition by PAD-S. 3\% preformed insulin fibrils were added as a template so that native insulin could bind to them and bypass the primary nucleation process. The samples were treated with PAD-S at 1:1 and 1:5 molar ratios and the aggregation kinetics were monitored by ThT assay (Figure 4A). The overall decrease in the ThT fluorescence intensity along with an extended lag phase of insulin aggregation indicated that PAD-S binds to native insulin and prevents both primary nucleation as well as elongation of insulin fibrillation.

To further validate the mode of inhibitory action of PAD-S, PAD-S was introduced at different time points $(2 \mathrm{~h}, 4 \mathrm{~h}$ and $7 \mathrm{~h}$ ) during insulin aggregation. PAD-S showed strong inhibition of insulin aggregation, and introduction after $2 \mathrm{~h}$ resulted in nearly complete inhibition of insulin aggregation. (Figure 4B). Similarly, introduction of PAD-S at $4 \mathrm{~h}$, which is at the start of log phase, arrested insulin aggregation. This signifies that PAD-S predominantly affects the primary nucleation and oligomerization events during insulin aggregation. We were surprised to find that introducing PAD-S at the end of $7 \mathrm{~h}$ resulted in reduction of the ThT fluorescence intensity to $\sim 60 \%$ of the maximum value. This is a striking result suggesting that $\mathrm{PAD}-\mathrm{S}$ could disintegrate mature fibrils (Figure 4B).

PAD-S disintegrates mature insulin fibrils. The previous finding led us to further probe whether PAD-S was successful in disintegrating mature insulin fibrils. As depicted in Figure 4C, PAD-S indeed disintegrated mature insulin fibrils in a time and dose dependent manner as seen in ThT kinetic assay. The final ThT fluorescence after disintegration of insulin fibrils at different doses of PAD-S after 1 hour of incubation is reported (Figure 4D).
PAD-S inhibits aggregation of insulin biosimilars. Next, we probed whether PAD-S could effectively inhibit aggregation of insulin biosimilars glargine and lispro which are currently under use for treatment of diabetes. ${ }^{36,37}$ Glargine is a slow acting insulin analog while lispro is a fast-acting insulin variant. ${ }^{38}$ Glargine consists of a glycine residue at the $\mathrm{C}$ terminal A-chain asparagine (N21G of A chain) and two extra arginine (T30R, R31 of $\mathrm{B}$ chain) residues at the $\mathrm{B}$-chain $\mathrm{C}$-terminal threonine. Insulin Lispro is made by reversal of amino acids at position 28 and 29 of the insulin B chain. ${ }^{39}$ It is a double mutant $\mathrm{P} 28 \mathrm{~K}, \mathrm{~K} 29 \mathrm{P}$ that has been developed to reduce dimerization, which results in its fast-acting property. ${ }^{40}$

Our aim was to understand whether PAD-S is effective against insulin variants as they have slight variation in their amino acid sequences in comparison with human insulin.
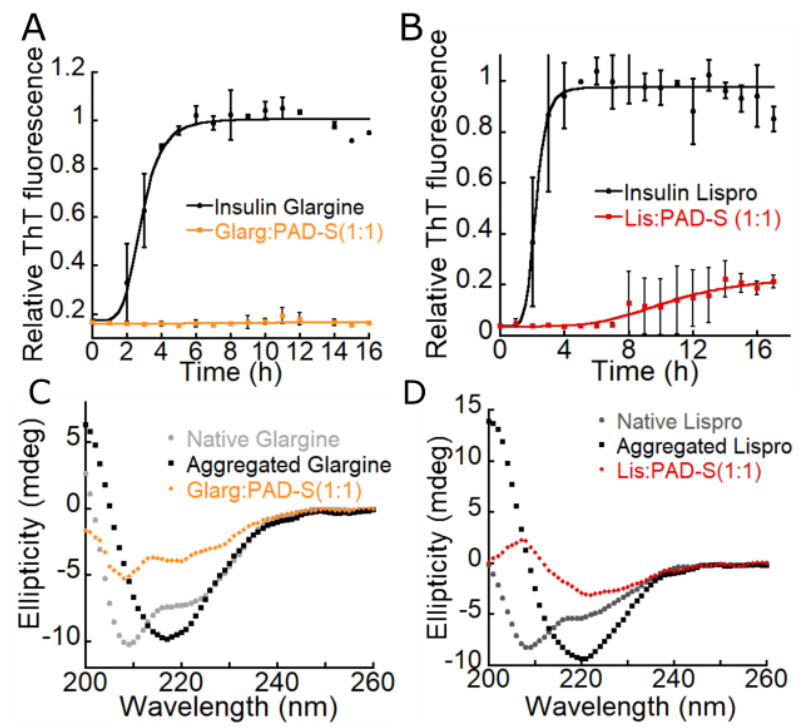

Figure 5. Fibrillation kinetics of insulin glargine (A), insulin lispro (B) in absence and presence of equimolar PAD-S (15 $\left.\mu \mathrm{M}, \mathrm{pH} 1.6,60^{\circ} \mathrm{C}\right)$. CD Spectra of insulin glargine (C), insulin lispro (D) in absence and presence of equimolar PAD-S (3.75 $\mu \mathrm{M})$ at the end of fibrillation. Data are average of three independent experiments. 

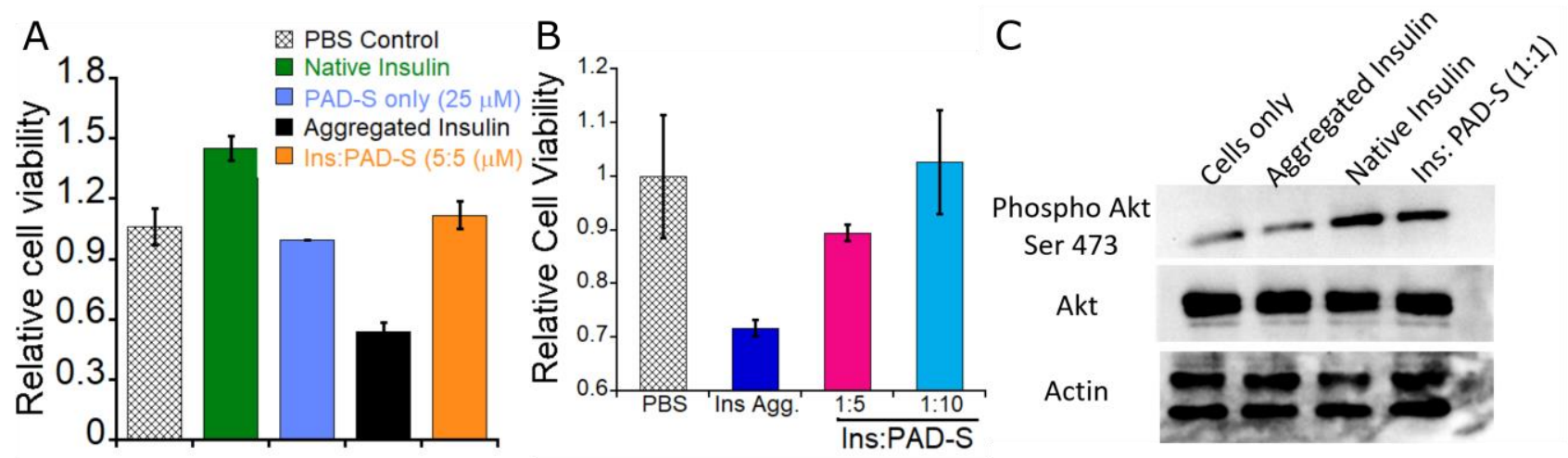

Figure 6. A) MTT assay showing toxicity of native insulin (5 $\mu \mathrm{M})$, insulin aggregates ( $5 \mu \mathrm{M})$, PAD-S (25 $\mu \mathrm{M})$ and insulin aggregated in presence of PAD-S at different molar ratio on HEK293T cells. The error bars indicate standard deviations from two independent experiments. B) MTT assay showing toxicity of insulin aggregates (5 $\mu \mathrm{M})$, mature insulin fibrils disintegrated in the presence of different concentrations of PAD-S on HEK293T cells. C) Western blotting to probe the phosphorylation of Akt (Ser 473) using PAD-S treated insulin. Akt and actin bands serve as controls. Data is average of three independent experiments.

This study will also delineate the importance of specific amino acid residues during PAD-S insulin interaction. At equimolar concentration, PAD-S completely inhibited aggregation of insulin glargine, while lispro aggregation was inhibited by $80 \%$ (Figure $5 \mathrm{~A}, \mathrm{~B}$ ). The weaker effect of PAD$\mathrm{S}$ on lispro suggests that PAD-S interacted specifically with Pro and Lys of insulin B chain to inhibit insulin aggregation. This was consistent with molecular docking studies which showed interaction of these residues with PAD-S in the insulin dimer. This further supports our hypothesis that PAD$S$ mainly stabilizes the insulin dimer, and that dimerization of insulin in presence of PAD-S significantly contributes to the anti-amyloid property of PAD-S. The inhibitory activity of PAD-S on insulin variants was corroborated by CD spectroscopy, which showed stabilization of the $\alpha$-helical structure of insulin glargine and bovine insulin, while some intermediate structures were observed in the case of insulin lispro (Figure 5C, D).

PAD-S protects HEK293T cell lines from amyloid induced toxicity of insulin. Since insulin amyloid fibrils are known to be cytotoxic, ${ }^{41,42}$ we checked whether treatment of insulin with PAD-S could prevent fibril induced toxicity to the cells. Cell viability was measured using MTT, which is converted to formazan in live cells and can be quantified by measuring its absorbance at $570 \mathrm{~nm}$. Human embryonic kidney cell line (HEK 293T) was treated with native insulin, insulin aggregates or insulin aggregated in the presence of PAD-S (5 $\mu \mathrm{M}$ each) (Figure 6A). PAD-S treated insulin showed cell viability similar to native insulin, compared to cells treated with insulin aggregates in which the cell viability was reduced to $\sim 50 \%$. This suggests that insulin treated with PAD-S is non-toxic to the cells mainly due to the absence of toxic insulin aggregates. PAD-S, up to a concentration of $25 \mu \mathrm{M}$ (maximum concentration of PAD-S used) was not significantly toxic to the cells under the same conditions (Figure 6A).

We also checked the cytotoxicity induced by the disintegrated fibrils generated after PAD-S treatment. The insulin samples obtained after disintegration were less toxic than mature fibrils as seen in MTT assay using HEK 293T cell line (Figure 6B), indicating that PAD-S was converting mature fibrils to alternative structures which are less toxic than mature fibrils.

PAD-S treated insulin is biologically active. Our in vitro results showed that PAD-S was successful in inhibiting insulin aggregation. We further examined the ability of aggregated insulin and insulin treated with PAD-S to activate insulin receptor based signaling. The activation of insulin dependent IR-signaling was probed through increase in phospho-Akt (Ser 473) levels using phospho-Akt specific antibodies. Control experiments were performed to determine the level of Akt and actin in the cells, which were found to be same in all the samples treated. We found the PAD-S treated insulin exhibited enhanced phospho-Akt levels comparable to native bioactive insulin, whereas untreated cells or cells treated with aggregated insulin showed basal pAkt levels (Figure 6C). This experiment suggests that PAD$\mathrm{S}$ treated insulin was bioactive and successfully activated insulin receptor signaling, thereby generating enhanced pAkt levels similar to native insulin.

\section{CONCLUSION}

In summary, we report the rational design of a small molecule called PAD-S based on our earlier reported insulin aggregation inhibitors. PAD-S acts as a chemical chaperone by preserving the native structure of insulin, and completely preventing aggregation of insulin and its biosimilars under harsh conditions. The sulfonate group attached to PAD-S makes it highly soluble in aqueous media, thereby facilitating its use in insulin formulations. PAD-S is non-toxic, and protects cells from insulin amyloid induced cytotoxicity. Molecular docking and mechanistic investigations lead to the conclusion that PAD-S mainly inhibits the primary nucleation and oligomerization during insulin aggregation by binding to monomers or dimers in the amyloid prone B11B17 residues of insulin. Furthermore, PAD-S was found to disintegrate mature insulin fibrils to non-toxic smaller fragments. PAD-S can thus be a valuable lead for regulating insulin fibrillation in commercial formulations.

\section{MATERIALS AND METHODS}


Materials. The reagents and solvents for chemical synthesis were purchased from commercial chemical suppliers. Recombinant human insulin was from MP Biomedicals. The following antibodies and reagents were purchased commercially from the sources mentioned: anti-phospho Akt (Ser473) antibody (4058, rabbit, Cell Signalling), anti-Akt (pan) antibody (4691, rabbit, Cell Signalling), and anti-actin antibody (mouse, BD Transduction Laboratories).

Insulin fibrillation. A stock of recombinant human insulin was prepared in $25 \mathrm{mM} \mathrm{HCl}$ containing $0.1 \mathrm{M} \mathrm{NaCl}(\mathrm{pH} \sim$ 1.6). The stock was diluted to $0.087 \mathrm{mg} / \mathrm{ml}(15 \mu \mathrm{M})$, and incubated at $60^{\circ} \mathrm{C}$ in a thermomixer with agitation of 300-400 rpm to promote fibrillation.

Thioflavin T (ThT) assay. ThT stock solution $(20 \mu \mathrm{M})$ was prepared in milliQ water. The aggregating insulin solution $(25 \mu \mathrm{l})$, (either alone or treated with given concentration of PAD-S) was pipetted out at specified time intervals, and added to $175 \mu \mathrm{l}$ of ThT solution. ThT fluorescence was measured using BioTek Cytation 1 Plate Reader. The excitation wavelength of ThT was $440 \mathrm{~nm}$ and the emission was $485 \mathrm{~nm}$.

Native PAGE. The aggregated insulin solutions $(30 \mu \mathrm{M}$, in the absence or presence of PAD-S) were centrifuged at 5000 $\mathrm{rpm}$ for $5 \mathrm{~min}$. The supernatant was analyzed by $15 \%$ native PAGE in tris-glycine running buffer $(\mathrm{pH} 8.8)$ at $100 \mathrm{~V}, 80 \mathrm{~A}$ for $1.5 \mathrm{~h}$. The protein was visualized using Coomassie brilliant blue stain. The gels were imaged using GeneSys software in GBOX (Syngene). Bands were quantified using Gene Tools software. ${ }^{22}$

Circular Dichroism. CD spectra were recorded for untreated insulin $(3.75 \mu \mathrm{M})$ [human recombinant and insulin variants] and those incubated with varying concentrations of PAD-S using a Jasco J-815 CD Spectrophotometer (JASCO, Japan) at room temperature. The following parameters were used: $1 \mathrm{~nm}$ bandwidth with $100 \mathrm{~nm} / \mathrm{min}$ scanning rate and data pitch of $0.5 \mathrm{~nm}$. Three accumulations were taken for each sample, and an average of at least 4 independent readings were plotted using Kaleidagraph software (Synergy, USA).

Transmission Electron Microscopy (TEM). $10 \mu \mathrm{l}$ of $1 \%$ (w/v) phosphotungstic acid solution was added to $10 \mu \mathrm{l}$ of insulin solution $(15 \mu \mathrm{M}$, alone or after incubation with PADS) for negative staining for $30 \mathrm{~min}$ at room temperature. The sample $(10 \mu \mathrm{l})$ was plated on Formavar protected carbon coated 400 mesh $\mathrm{Cu}$ grids (Ted Pella Inc.) and allowed to adsorb for 5 minutes. The excess sample was removed using a tissue paper, and the grid was dried in a vacuum desiccator. TEM analysis was performed on the dried samples using FEI Talos $200 \mathrm{~S}$ system equipped with a $200 \mathrm{kV}$ Field Emission Gun (FEG).

Protein-ligand docking. Molecular docking was performed to evaluate the binding site of PAD-S with insulin using Autodock 4.0. The 3-D structure of PAD-S was prepared using ChemDraw, and energy minimization was performed using Chem3D software. The native structure of insulin from RCSB Protein Data Bank (PDB: 1GUJ) comprised of insulin dimer consisting of two monomers (Chains A/B and identical C/D) was used for docking. Either A/B or C/D was removed to make the insulin monomer which was used for docking purpose. Grid size along X-, Y- and Z- axes were fixed as 85,85 , and 85 respectively, with grid spacing 0.375
$\AA$ A for the monomer and 119, 97, 126 with grid spacing of $0.375 \AA$ for the insulin dimer. Blind docking was performed to identify the ligand binding site. Both protein and the ligand were prepared by applying Gasteiger charges. All other parameters were set to default. The results were analyzed and figures were prepared using Discovery Studio Visualizer Software, Version 4.0 (2012).

Titration of PAD-S with insulin. Human recombinant insulin $(5 \mu \mathrm{M})$ was titrated with increasing concentration of PAD-S (0-100 $\mu \mathrm{M})$. The samples were allowed to equilibrate for $30 \mathrm{~min}$ before each reading. Tyrosine (Excitation: 275 $\mathrm{nm})$ fluorescence emission was measured between 290$350 \mathrm{~nm}$. Tyrosine fluorescence at $303 \mathrm{~nm}$ was plotted for each concentration of PAD-S. The $\mathrm{K}_{d}$ was determined using the equation:

$\mathrm{F}=\mathrm{F}_{\max } \frac{\mathrm{K}_{\mathrm{d}}+[\mathrm{Ins}]+[\mathrm{PAD}-\mathrm{S}]-\sqrt{\left(\mathrm{K}_{\mathrm{d}}+[\mathrm{Ins}]+[\mathrm{PAD}-\mathrm{S}]\right)^{2}-4[\mathrm{Ins}][\mathrm{PAD}-\mathrm{S}]}}{2[\mathrm{Ins}]}$

$F_{\max }$ is the maximum fluorescence intensity at saturating PAD-S concentration and $\mathrm{K}_{d}$ is the equilibrium dissociation constant of the Ins-PAD-S complex.

Seeded insulin fibrillation. Seeded fibrillation was carried out using fibrils obtained by the aggregation of insulin solutions. $3 \%$ seeds $(\mathrm{v} / \mathrm{v})$ were added to native insulin, either in the absence or presence of PAD-S before the onset of fibrillation. ThT measurements were performed as described above.

Insulin fibrillation kinetics with time dependent addition of PAD-S. PAD-S $(15 \mu \mathrm{M})$ was added at different time points $(0,2,4,7 \mathrm{~h})$ to aggregating insulin solutions. The ThT measurements were performed as described above.

Competition experiment with LVEALYL. To study the competitive binding between LVEALYL and PAD-S to insulin, we incubated $15 \mu \mathrm{M}$ of insulin with or without the peptide at a ratio of 1:0.25 (Ins: LVEALYL) and with equimolar concentration of PAD-S compared to insulin. The solutions were incubated using standard conditions of aggregation as mentioned above. At every hour, aggregating insulin solution was pipetted out and ThT fluorescence measurements were performed.

Cell viability assay. HEK-293T cells were grown in DMEM media having $10 \%$ fetal bovine serum (FBS), using a humified atmosphere $\left(5 \% \mathrm{CO}_{2}\right)$ at $37^{\circ} \mathrm{C}$. 5000 cells were seeded in 96-well plates per well and incubated for $24 \mathrm{~h}$. The media was removed and the cells were treated with $100 \mu \mathrm{L}$ of the serum media containing $5 \mu \mathrm{M}$ aggregated insulin solution containing a mixture of oligomers and fibrils (either alone or PAD-S treated) and incubated for $24 \mathrm{~h}$. The cell viability was measured using an MTT assay (Sigma-Aldrich). $100 \mu \mathrm{L}$ of $5 \mathrm{mg} / \mathrm{ml}$ MTT solution diluted in media, was added to each well and incubated for $4 \mathrm{~h}$. The media was removed and $150 \mu \mathrm{L}$ of DMSO was added into each well to dissolve the formazan crystals. The absorption was measured at 570 nm using BioTek Cytation 1 Plate Reader (USA).

Cell culture for Akt phosphorylation. To check cell-based activity of insulin, 50,000 HEK-293T cells were grown in DMEM media till about $70 \%$ confluency as described above. The cells were then serum starved for $4 \mathrm{~h}$ in no serum DMEM media. Then the cells were treated with only media, native insulin, untreated aggregated insulin or PAD-S treated insulin at $100 \mathrm{nM}$ concentration. For untreated aggregated insulin, after complete fibrillation, the fibrils were 
settled by a quick spin and the resultant supernatant was taken and applied to the cells. After 40 minutes of treatment, the cells were lysed using lysis buffer $(50 \mathrm{mM}$ Tris $\mathrm{pH}$ 7.5, $100 \mathrm{mM} \mathrm{NaCl}, 1 \mathrm{mM}$ EDTA, 1\% Triton-X 100) along with $1 \mathrm{X}$ protease inhibitor tablets (Roche) in ice cold condition. The lysed cells were collected in respective tubes, centrifuged at $13000 \mathrm{rpm}$ at $4{ }^{\circ} \mathrm{C}$ for $1-2$ minutes to separate the pellet. The supernatant was mixed with $2 X$ SDS running dye and the samples were boiled at $100{ }^{\circ} \mathrm{C}$ for 10 minutes. After a quick spin, the samples were run on SDS-PAGE.

Western Blotting. $15 \mu$ l of the sample was loaded on $15 \%$ SDS-PAGE in tris-glycine running buffer ( $\mathrm{pH}$ 8.8). The run was performed at $130 \mathrm{~V}$ for 120 minutes and $100 \mathrm{~A}$, after which the samples were transferred to a Millipore polyvinylidene difluoride (PVDF) membrane (IPVH000-10) at 90 $\mathrm{V}$ for 120 minutes at $4{ }^{\circ} \mathrm{C}$ using Transfer buffer (48 mM Tris base, $39 \mathrm{mM}$ Glycine, 20\% Methanol, $0.375 \%$ SDS). The membranes were blocked using 3\% Non-fat milk in $1 \mathrm{X}$ TBST $(20 \mathrm{mM}$ Tris- $\mathrm{HCl} \mathrm{pH} 7.5,150 \mathrm{mM} \mathrm{NaCl}, 0.1 \%$ Tween$20)$. The membranes were incubated with the desired primary antibody at $4{ }^{\circ} \mathrm{C}$ overnight. They were then washed with $1 \mathrm{X}$ TBST for 3 times, 5 minutes each. The membranes were subsequently incubated with respective secondary antibodies for $1.5 \mathrm{~h}$ at room temperature. They were then developed using ECL solution containing 1:1 Luminol solution (GE Healthcare) and peroxide solution (GE Healthcare). The membranes were then visualized using GeneSys software in GBOX (Syngene).

\section{AUTHOR INFORMATION}

\section{Corresponding Author \\ * ishu@iiserb.ac.in.}

\section{Author Contributions}

[+] These authors contributed equally

Notes

The authors declare no conflict of financial interest.

\section{ACKNOWLEDGMENT}

We thank IISER Bhopal, and SERB for financial support, and DST-FIST facility at IISER Bhopal for TEM images. YMG thanks UGC for a Senior Research Fellowship.

\section{REFERENCES}

(1) De Meyts, P.; Whittaker, J. Structural Biology of Insulin and IGF1 Receptors: Implications for Drug Design. Nat. Rev. Drug Discov. 2002, 1 (10), 769-783.

(2) Brereton, M. F.; Rohm, M.; Ashcroft, F. M. $\beta$-Cell Dysfunction in Diabetes: A Crisis of Identity? Diabetes, Obes. Metab. 2016, 18 (S1), 102-109.

(3) Sluzky, V.; Tamada, J. A.; Klibanov, A. M.; Langer, R. Kinetics of Insulin Aggregation in Aqueous Solutions upon Agitation in the Presence of Hydrophobic Surfaces. Proc. Natl. Acad. Sci. 1991, 88 (21), 9377.

(4) Nielsen, L.; Khurana, R.; Coats, A.; Frokjaer, S.; Brange, J.; Vyas, S.; Uversky, V. N.; Fink, A. L. Effect of Environmental Factors on the Kinetics of Insulin Fibril Formation: Elucidation of the Molecular Mechanism. Biochemistry 2001, 40 (20), 6036-6046.

(5) Ratner, R. E.; Phillips, T. M.; Steiner, M. Persistent Cutaneous Insulin Allergy Resulting from High-Molecular-Weight Insulin Aggregates. Diabetes 1990, 39 (6), 728-733.
(6) Robbins, D. C.; Mead, P. M. Free Covalent Aggregates of Therapeutic Insulin in Blood of Insulin-Dependent Diabetics. Diabetes 1987, 36 (2), 147-151.

(7) Störkel, S.; Schneider, H. M.; Müntefering, H.; Kashiwagi, S. Iatrogenic, Insulin-Dependent, Local Amyloidosis. Lab. Invest. 1983, 48 (1), 108-111.

(8) Kourie, J. I.; Henry, C. L. Ion Channel Formation and Membrane-Linked Pathologies of Misfolded Hydrophobic Proteins: The Role of Dangerous Unchaperoned Molecules. Clin. Exp. Pharmacol. Physiol. 2002, 29 (9), 741-753.

(9) Gong, H.; He, Z.; Peng, A.; Zhang, X.; Cheng, B.; Sun, Y.; Zheng, L.; Huang, K. Effects of Several Quinones on Insulin Aggregation. Sci. Rep. 2014, 4, 5648.

(10) Jayamani, J.; Shanmugam, G.; Azhagiya Singam, E. R. Inhibition of Insulin Amyloid Fibril Formation by Ferulic Acid\{,\} a Natural Compound Found in Many Vegetables and Fruits. RSC Adv. 2014, 4 (107), 62326-62336.

(11) Jayamani, J.; Shanmugam, G. Gelatin as a Potential Inhibitor of Insulin Amyloid Fibril Formation. ChemistrySelect 2016, 1 (15), 4463-4471.

(12) Vilasi, S.; Iannuzzi, C.; Portaccio, M.; Irace, G.; Sirangelo, I. Effect of Trehalose on W7FW14F Apomyoglobin and Insulin Fibrillization: New Insight into Inhibition Activity. Biochemistry 2008, 47 (6), 1789-1796.

(13) Wang, J. B.; Wang, Y. M.; Zeng, C. M. Quercetin Inhibits Amyloid Fibrillation of Bovine Insulin and Destabilizes Preformed Fibrils. Biochem. Biophys. Res. Commun. 2011, 415 (4), 675-679.

(14) Hee, L. H.; Su, C. T.; C., L. S. J.; Wha, L. J.; Junghong, P.; Ho, K. Y.; Jong, K. W.; Kimoon, K.; I., K. H. Supramolecular Inhibition of Amyloid Fibrillation by Cucurbit[7]Uril. Angew. Chemie Int. Ed. 2014, 53 (29), 7461-7465.

(15) Shinde, M. N.; Barooah, N.; Bhasikuttan, A. C.; Mohanty, J. Inhibition and Disintegration of Insulin Amyloid Fibrils: A Facile Supramolecular Strategy with p-Sulfonatocalixarenes. Chem. Commun. 2016, 52 (14), 2992-2995.

(16) Shinde, M. N.; Khurana, R.; Barooah, N.; Bhasikuttan, A. C.; Mohanty, J. Sulfobutylether- $\beta$-Cyclodextrin for Inhibition and Rupture of Amyloid Fibrils. J. Phys. Chem. C 2017, 121 (36), 2005720065.

(17) Banerjee, V.; Kar, R. K.; Datta, A.; Parthasarathi, K.; Chatterjee, S.; Das, K. P.; Bhunia, A. Use of a Small Peptide Fragment as an Inhibitor of Insulin Fibrillation Process: A Study by High and Low Resolution Spectroscopy. PLoS One 2013, 8 (8), e72318.

(18) Ratha, B. N.; Ghosh, A.; Brender, J. R.; Gayen, N.; Ilyas, H.; Neeraja, C.; Das, K. P.; Mandal, A. K.; Bhunia, A. Inhibition of Insulin Amyloid Fibrillation by a Novel Amphipathic Heptapeptide: MECHANISTIC DETAILS STUDIED BY SPECTROSCOPY IN COMBINATION WITH MICROSCOPY. J. Biol. Chem. 2016, 291 (45), 23545-23556.

(19) Ratha, B. N.; Kar, R. K.; Kalita, S.; Kalita, S.; Raha, S.; Singha, A.; Garai, K.; Mandal, B.; Bhunia, A. Sequence Specificity of Amylin-Insulin Interaction: A Fragment-Based Insulin Fibrillation Inhibition Study. Biochim. Biophys. Acta - Proteins Proteomics 2019, 1867 (4), 405-415.

(20) Siddiqi, M. K.; Alam, P.; Iqbal, T.; Majid, N.; Malik, S.; Nusrat, S.; Alam, A.; Ajmal, M. R.; Uversky, V. N.; Khan, R. H. Elucidating the Inhibitory Potential of Designed Peptides Against Amyloid Fibrillation and Amyloid Associated Cytotoxicity. Front. Chem. 2018, $6,311$.

(21) Zheng, Q.; Lazo, N. D. Mechanistic Studies of the Inhibition of Insulin Fibril Formation by Rosmarinic Acid. J. Phys. Chem. B 2018, 122 (8), 2323-2331.

(22) Klostermeyer, H.; Humbel, R. E. The Chemistry and Biochemistry of Insulin. Angew. Chemie Int. Ed. English 1966, 5 (9), 807-822.

(23) Ivanova, M. I.; Sievers, S. A.; Sawaya, M. R.; Wall, J. S.; Eisenberg, D. Molecular Basis for Insulin Fibril Assembly. Proc. Natl. Acad. Sci. U. S. A. 2009, 106 (45), 18990-18995.

(24) Mishra, N. K.; Joshi, K. B.; Verma, S. Inhibition of Human and Bovine Insulin Fibril Formation by Designed Peptide Conjugates. Mol. Pharm. 2013, 10 (10), 3903-3912. 
(25) Das, A.; Gangarde, Y. M.; Tomar, V.; Shinde, O.; Upadhyay, T.; Alam, S.; Ghosh, S.; Chaudhary, V.; Saraogi, I. Small-Molecule Inhibitor Prevents Insulin Fibrillogenesis and Preserves Activity. Mol. Pharm. 2020, 17 (6), 1827-1834.

(26) Gangarde, Y. M.; Das, A.; Ajit, J.; Saraogi, I. Synthesis and Evaluation of Arylamides with Hydrophobic Side Chains for Insulin Aggregation Inhibition. Chempluschem 2021, 86 (5), 750-757.

(27) Giger, K.; Vanam, R. P.; Seyrek, E.; Dubin, P. L. Suppression of Insulin Aggregation by Heparin. Biomacromolecules 2008, 9 (9), 2338-2344.

(28) Hong, Y.; Meng, L.; Chen, S.; Leung, C. W. T.; Da, L.-T.; Faisal, M.; Silva, D.-A.; Liu, J.; Lam, J. W. Y.; Huang, X.; et al. Monitoring and Inhibition of Insulin Fibrillation by a Small Organic Fluorogen with Aggregation-Induced Emission Characteristics. J. Am. Chem. Soc. 2012, 134 (3), 1680-1689.

(29) Owczarz, M.; Arosio, P. Sulfate Anion Delays the Self-Assembly of Human Insulin by Modifying the Aggregation Pathway. Biophys. J. 2014, 107 (1), 197-207.

(30) Groenning, M.; Norrman, M.; Flink, J. M.; van de Weert, M.; Bukrinsky, J. T.; Schluckebier, G.; Frokjaer, S. Binding Mode of
Thioflavin T in Insulin Amyloid Fibrils. J. Struct. Biol. 2007, 159 (3), 483-497.

(31) Hua, Q.; Weiss, M. A. Mechanism of Insulin Fibrillation: THE STRUCTURE OF INSULIN UNDER AMYLOIDOGENIC CONDITIONS RESEMBLES A PROTEIN-FOLDING INTERMEDIATE . J. Biol. Chem. 2004, 279 (20), 21449-21460.

(32) Brange, J.; Andersen, L.; Laursen, E. D.; Meyn, G.; Rasmussen, E. Toward Understanding Insulin Fibrillation. J. Pharm. Sci. 1997, 86 (5), 517-525.

(33) Uversky, V. N.; Garriques, L. N.; Millett, I. S.; Frokjaer, S.; Brange, J.; Doniach, S.; Fink, A. L. Prediction of the Association State of Insulin Using Spectral Parameters. J. Pharm. Sci. 2003, 92 (4), 847-858.

(34) Nielsen, L.; Frokjaer, S.; Brange, J.; Uversky, V. N.; Fink, A. L. Probing the Mechanism of Insulin Fibril Formation with Insulin Mutants. Biochemistry 2001, 40 (28), 8397-8409.

(35) Arosio, P.; Vendruscolo, M.; Dobson, C. M.; Knowles, T. P. J. Chemical Kinetics for Drug Discovery to Combat Protein Aggregation Diseases. Trends Pharmacol. Sci. 2014, 35 (3), 127-135 